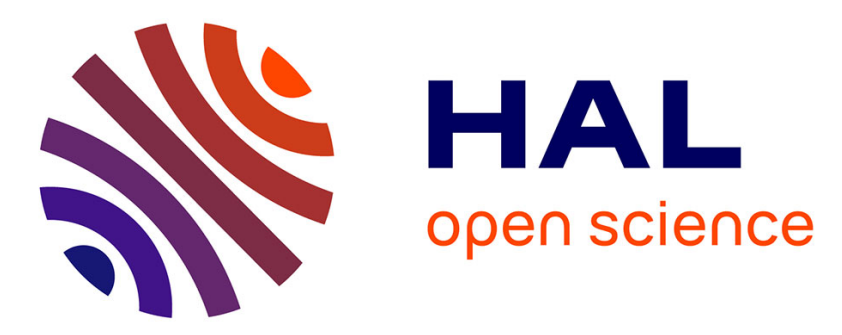

\title{
Surface tension and deformations of membrane structures : relation to two-dimensional phase transitions
}

\author{
F. Brochard, P.-G. de Gennes, P. Pfeuty
}

\section{To cite this version:}

F. Brochard, P.-G. de Gennes, P. Pfeuty. Surface tension and deformations of membrane structures: relation to two-dimensional phase transitions. Journal de Physique, 1976, 37 (10), pp.1099-1104. 10.1051/jphys:0197600370100109900 . jpa-00208507

\section{HAL Id: jpa-00208507 https://hal.science/jpa-00208507}

Submitted on 1 Jan 1976

HAL is a multi-disciplinary open access archive for the deposit and dissemination of scientific research documents, whether they are published or not. The documents may come from teaching and research institutions in France or abroad, or from public or private research centers.
L'archive ouverte pluridisciplinaire HAL, est destinée au dépôt et à la diffusion de documents scientifiques de niveau recherche, publiés ou non, émanant des établissements d'enseignement et de recherche français ou étrangers, des laboratoires publics ou privés. 


\title{
SURFACE TENSION AND DEFORMATIONS OF MEMBRANE STRUCTURES : RELATION TO TWO-DIMENSIONAL PHASE TRANSITIONS
}

\author{
F. BROCHARD, P. G. DE GENNES and P. PFEUTY \\ Laboratoire de Physique des Solides (*), Université Paris-Sud, 91405 Orsay, France
}

(Reçu le 11 mars 1976, accepté le 13 mai 1976)

Résumé. - On analyse les déformations d'une membrane flexible pour deux types de systèmes :

1) Systèmes fermés (ex. : membrane d'une cellule biconcave de globule rouge) où le nombre de constituants chimiques de la membrane est fixé, mais la surface $S$ est ajustable.

2) Systèmes ouverts (ex. : film formé par une bicouche) où les molécules sont en contact avec un réservoir.

Dans le premier cas, la tension de surface est nulle. Les fluctuations sont par conséquent très larges et limitées seulement par certains termes anharmoniques. Nous montrons que ces termes peuvent être calculés exactement. Ce problème est relié au modèle sphérique des transitions de phase. On retrouve pour la diffusion de la lumière les lois calculées dans un modèle harmonique plus simple (construit récemment par l'un d'entre nous).

Dans le second cas, il y a une tension de surface finie et les fluctuations sont très réduites. Ce résultat est en accord avec des mesures récentes de diffusion de la lumière par des films noirs obtenus par Grabowski et Cowen.

Abstract. - We analyse the thermal fluctuations of two types of flexible membranes :

1) Closed systems (such as the membrane of a biconcave red blood cell) where the chemical constituents of the membrane are fixed in number, but the membrane surface $S$ is adjustable.

2) Open systems (such as bilayer films) where constituent molecules can be exchanged with a reservoir (at the outer surface of the rim).

For the first case, the surface tension is zero; fluctuations are then very large, and limited only by certain anharmonic terms. We show that these terms can be analysed exactly - the problem being related to the spherical model of phase transitions. Although the anharmonic terms are strong, they do not modify the laws derived for the light scattering in the simpler harmonic model constructed earlier by one of us [1].

For the second case, there is a finite surface tension, and the fluctuations are much less pathological. This seems to agree with recent measurements by inelastic light scattering on black films by Grabowski and Cowen.

1. Introduction. - Thin, flexible, membranes are realized in particular with the bilayer films of Mueller [2] (Fig. 1). They are also found at the surface of biological cells : an example of primary interest is the red blood cell [3] shown in figure 2. For many mechanical or optical studies, it is of interest to understand the long range deformations of these thin structures. At first sight, it appears natural to assume that there is a surface tension which tends to limit the deformations. However, upon reflection, membranes are qualitatively different from a simple fluid/fluid interface : in the latter system an increase of surface is realized by bringing more molecules from both fluids towards the surface. But in a red blood cell, for ins-

$\left(^{*}\right)$ Laboratoire associé au C.N.R.S.

LE JOURNAL DE PHYSIQUE. - T. 37, No 10, OCTOBRE 1976 tance, the number of molecules participating in the membrane is essentially fixed. There is thus a real question as to the existence of a surface tension in membrane systems : it was raised (but not solved !) in a previous paper by Papoular and one of us [4].

More recently, Lennon and one of us [1] reported experiments on the flicker phenomenon in red blood cells and proposed an interpretation for them. The key to the interpretation was

a) to assume zero surface tension for unswollen cells,

b) to treat the system in a small motion (harmonic) approximation. The results were in plausible agreement with various scaling laws extracted from the data. However, one serious problem remained : the anharmonic terms, when examined, appeared strong, 


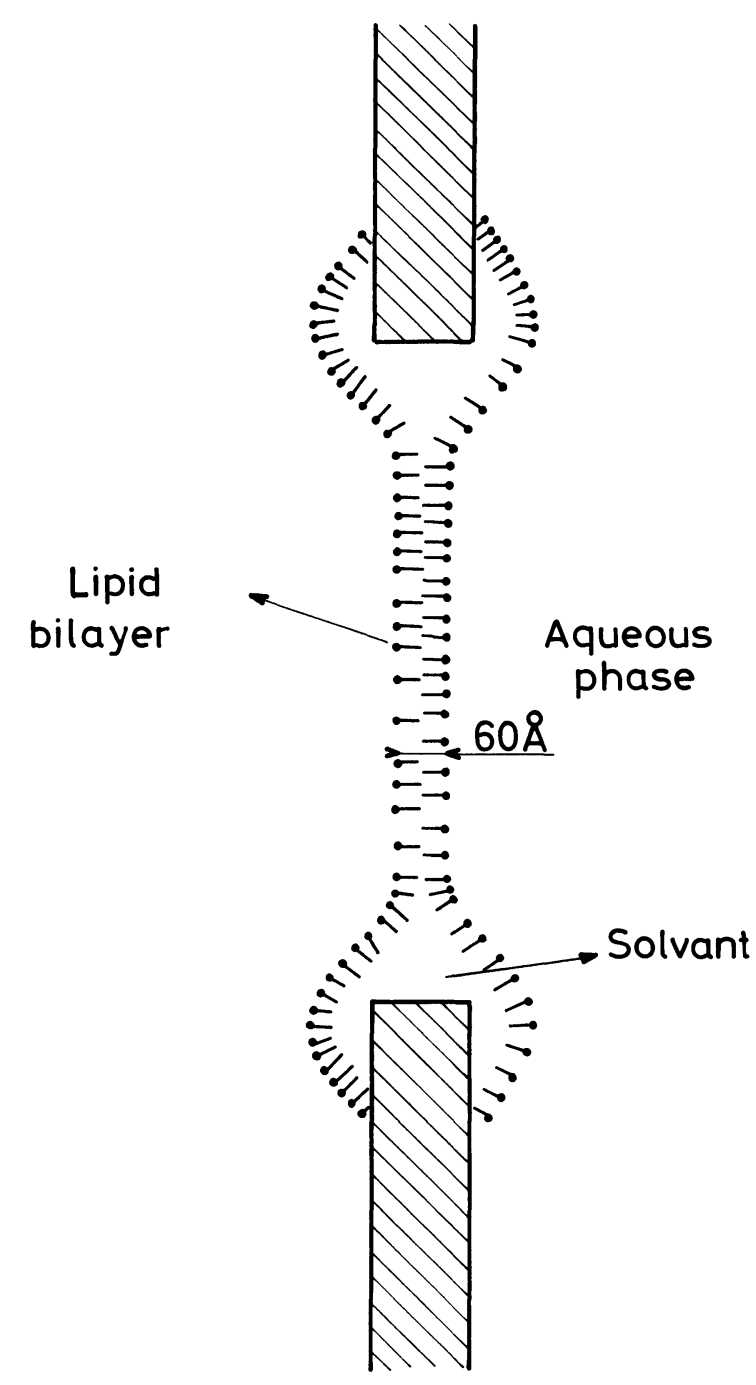

FIG. 1. - Open system, the lipid bilayer exchanges molecules with the outer ring.

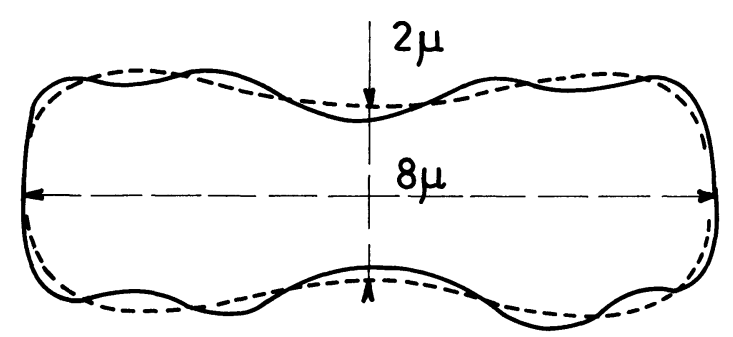

Fig. 2. - Closed system : human erythrocyte. Dotted line : equilibrium biconcave discoidal shape of a normal erythrocyte. Full line : ripple deformation of the $\mathrm{RBC}$ membrane.

and could be dangerous in a similar manner to the 4th order terms in the Landau-Ginsburg free energy of a system near a critical point [5].

Unfortunately, the discussion of the anharmonic terms in ref. [1] was based on an incorrect analytic form. The aims of the present paper are :

1) to correct this, and discuss fully the anharmonic effects for the red blood cell case. It turns out that with the correct form, the problem is entirely soluble, and that the conclusions of the simple harmonic model remain valid (section 2);

2) to discuss the fluctuations of black films : here, we are dealing with an open system, as is clear from figure 1. A change in the shape of the film can be accompanied by an increase in the number of lipids in the film : the situation is thus much closer to the fluid/ fluid interface, and indeed we shall see that a finite (although small) surface tension is maintained in this case (section 3).

Our emphasis is constantly on principles, not on numbers. In particular we make no attempt to include in our discussion the real shape of a red blood cell, and we do not predict the actual magnitude of any of the coefficients involved.

\section{Deformations of closed membranes.}

2.1 Volume AND SURFACE VARIABLES. - Our typical example is a red blood cell with surface area $S$ and internal volume $V$. The following properties are essential :

(i) The membrane of surface $S$ is made of a fixed number of constituent molecules (lipid + protein). The local surface density of these constituents will be denoted by $\rho$ and we may write

$$
\int \rho \mathrm{d} \Sigma \equiv \bar{\rho} S=\rho_{0} S_{0}
$$

where $\mathrm{d} \Sigma$ is a surface element, $S$ is the deformed surface; $S_{0}$ and $\rho_{0}$ are the unperturbed surface and density. The density $\bar{\rho}$ is the average of $\rho$ in the deformed state.

(ii) The red blood cell is unswollen in the following sense : the internal volume $V$ is smaller than a critical value $V_{c}$ which would correspond to a sphere of area $S_{0}$

$$
V_{\mathrm{c}}=\left(\frac{S_{0}}{4 \pi}\right)^{3 / 2} \frac{4 \pi}{3}
$$

when $V<V_{\mathrm{c}}$ the variables $S_{0}$ and $V$ are independent, and the overall free energy $F$ of the system must be minimal with respect to both of them. This implies that

$$
r=\left.\frac{\partial F}{\partial S}\right|_{S_{0}}=0
$$

The surface tension $r$ must vanish in this case, as explained in ref. [1].

2.2 Idealisation OF THE SHAPE. - From now on we shall ignore the real shape of the red blood cell, and substitute for it a sheet lying near the $(x y)$ plane, and described by a vertical displacement $u(x y)$.

The surface $S_{0}$ is replaced by a square $(0<x<L$ and $0<y<L$ ) of area $S_{0}=L^{2}$. To imitate the fact 
that $S_{0}$ has no edge, but is closed on itself, we shall impose periodic boundary conditions

$$
u(x, y)=u(x+L, y)=u(x, y+L) .
$$

This will allow for a simple Fourier analysis of the modes

$$
\begin{gathered}
u_{q}=\frac{1}{2 \pi} \int \mathrm{d} x \mathrm{~d} y u(x y) \mathrm{e}^{-i\left(q_{\mathrm{x}} x+q_{y} y\right)} \\
u(x y)=\sum_{q} u_{q} \mathrm{e}^{i\left(q_{\mathrm{x}} x+q_{y} y\right)} \cong \frac{1}{2 \pi} \int \mathrm{d} q_{x} \mathrm{~d} q_{y} u_{q} \mathrm{e}^{i\left(q_{\mathrm{x}} x+q_{y} y\right)}
\end{gathered}
$$

where $q=0$ will be excluded (to maintain the equivalent of a constant volume inside the cell).

2.3 Structure OF THE Elastic ENERGy. - The elastic energy of the distorted membrane has the following contributions

a) A bare surface tension term

$$
f_{\mathrm{s}}=r_{0}\left(S-S_{0}\right) \text {. }
$$

The reason for maintaining this $r_{0}$ is the following; after inclusion of the fluctuation effects, we shall find that the complete surface tension $\tilde{r}$ differs from $r_{0}$

$$
\tilde{r}=r_{0}+\delta r \text {. }
$$

As explained in [1], it is this total coefficient $r$ which must be set equal to 0 .

b) The elastic energy due to changes in the local density $\rho$ of the membrane is

$$
\begin{aligned}
f_{\mathrm{d}} & =\int \mathrm{d} x \mathrm{~d} y \frac{1}{2} E\left(\frac{\rho-\rho_{0}}{\rho_{0}}\right)^{2} \\
& =S \frac{1}{2} E\left(\frac{\bar{\rho}-\rho_{0}}{\rho_{0}}\right)^{2}+\frac{1}{2} E \int \mathrm{d} x \mathrm{~d} y\left(\frac{\rho-\bar{\rho}}{\rho_{0}}\right)^{2} .
\end{aligned}
$$

The last term in this expression describes the density fluctuations of non zero wave vector inside the film, and is not different from what we have in an unperturbed film : it is thus completely decoupled from the ripples in which we are interested, and can be omitted in what follows. This leads to

$$
\begin{aligned}
f_{\mathrm{d}} & =\frac{1}{2} E \frac{\left(S-S_{0}\right)^{2}}{S} \\
& =\frac{E}{2 S}\left(\int \mathrm{d} x \mathrm{~d} y \frac{1}{2}(\nabla u)^{2}\right)^{2}
\end{aligned}
$$

which differs from the form used in ref. [1]. The incorrect procedure used in ref. [1] amounted to assuming that the molecules had no horizontal motion when the displacement $u(x y)$ was applied. This then imposed a change in the local density

$$
\frac{\rho-\rho_{0}}{\rho_{0}}=\frac{1}{2}(\nabla u)^{2}
$$

and led to the form

$$
f_{\mathrm{d}}=\frac{1}{2} E \int \mathrm{d} x \mathrm{~d} y\left(\frac{1}{2}(\nabla u)^{2}\right)^{2} \quad \text { (incorrect) }
$$

In actual fact, the molecules do move horizontally to minimize the average of $(\rho-\bar{\rho})^{2}$, and this leads to $(2.10)$.

In addition to the two-dimensional compressional energy (2.8) we might have to include another term, related to shear deformation : this will occur if the membrane is not a liquid, but rather a rubber-like material, as emphasized by Evans [6]. This however will not affect our discussion, and is omitted here for simplicity.

c) Another essential contribution to the elasticity is the curvature energy. - The importance of this term for lipid bilayers and red blood cell membranes has been emphasized by Helfrich [7] and by others $\left({ }^{1}\right)$. This energy is of the form

$$
f_{\mathrm{c}}=\frac{1}{2} K \int \mathrm{d} x \mathrm{~d} y\left(C-C_{0}\right)^{2}
$$

where $K$ is a constant with the dimension of an energy, of order $10^{-13}$ ergs.

$C=\nabla^{2} u$ is the local curvature, and $C_{0}$ is the spontaneous curvature (present if the two sides of the membranes differ). The role of $C_{0}$ is important for understanding the biconcave form of real red blood cells; but for our idealised problem $C_{0}$ must be ignored.

2.4 FluCTUATION EFFECTS. - Having specified the free energy $f=f_{\mathrm{d}}+f_{\mathrm{e}}+f_{\mathrm{c}}$ for any deformation of the membrane, we can find the statistical weight for any conformation as exp $-f / T$ and obtain the partition function of the membrane by summing these weights over all possible shapes. It turns out that this problem is solvable exactly, even in the presence of the anharmonic terms (2.10). In the language of phase transitions, the membrane system is a realization of the two-dimensional spherical model for which there exists an extensive literature [8]. The analogy can be seen on Fourier transformation : defining

$$
m_{q}=|q| u_{q}
$$

the free energy $f$ is

$f=\frac{1}{2 \pi} \int \frac{1}{2}\left(K q^{2}+r\right) m_{q} m_{-q} \mathrm{~d}^{2} q+\frac{E}{8 S_{0}} I^{2}$

where

$$
I=\frac{1}{2 \pi} \int \mathrm{d}^{2} q m_{q} m_{-q} .
$$

Eq. (2.14) has indeed the structure associated with a one component spherical model. The main feature

(1) Gruler, H., private communication. 
is that the quantity $I$ is extensive (of order $S_{0}$ ) and has only weak relative fluctuations (of order $S_{0}^{-1 / 2}$ ). The calculation of the partition function $Z$ is classical and will be summarized only briefly here. We write

$$
\begin{aligned}
Z & =\int_{0}^{\infty} Z_{0}(r I) \exp \left(-\frac{v I^{2}}{T}\right) \mathrm{d} I \\
v & =\frac{E}{8 S_{0}}
\end{aligned}
$$

where $Z_{0}(r I)$ is the partition function calculated without the anharmonic terms but with the constraint that the integral in (2.15) has a fixed value $I$. It is easy to calculate the Laplace transform of $Z_{0}$

$$
\begin{aligned}
Z_{0}(r P) & =\int_{0}^{\infty} \mathrm{d} I \mathrm{e}^{-P I} Z_{0}(r I) \\
& =Z_{\mathrm{free}}(r+2 P T)
\end{aligned}
$$

where $Z_{\mathrm{free}}$ is the partition function calculated without the anharmonic terms and without any constraint. $Z_{\mathrm{free}}$ is the partition function of a gaussian system and is given by

$$
\log Z_{\mathrm{free}}(r)=\frac{1}{2} \sum_{q} \ln \left(\frac{T}{r+K q^{2}}\right) .
$$

Knowing $Z_{\text {free }}$ and $Z_{0}(r P)$ we can now return to $Z_{0}(r I)$

$$
Z_{0}(r, I)=\frac{1}{2 \pi i} \int_{-i \infty}^{i \infty} \mathrm{d} P \mathrm{e}^{P I} Z_{0}(r P)
$$

and finally to the complete partition function

$$
\begin{aligned}
Z=\frac{1}{2 \pi i} \int_{-i \infty}^{i \infty} \mathrm{d} P & Z_{\mathrm{free}}(r+2 P T) \times \\
& \times \int_{0}^{\infty} \mathrm{d} I \exp \left(P I-\frac{v I^{2}}{T}\right) .
\end{aligned}
$$

The last integrand has a peak at $I=T P / 2 v$ (linear in $\left.S_{0}\right)$ and a width of order $v^{-1 / 2}\left(\sim S_{0}^{1 / 2}\right)$. Thus the integral can be safely taken from $-\infty$ to $+\infty$ and gives const. $\exp \left(T P^{2} / 4 v\right)$.

The integral over $P$ can then be performed by saddle point methods : the saddle point condition is

$$
\frac{\partial}{\partial P} \ln Z_{\mathrm{free}}(r+2 P T)+\frac{P T}{2 v}=0
$$

or through $(2.18)$

$$
-\sum_{q} \frac{1}{r+2 P T+K q^{2}}+\frac{P}{2 v}=0 .
$$

This is an implicit equation for $P$. We prefer to write it in terms of a renormalized surface tension $\tilde{r}=r+2 P T$

$$
\tilde{r}=r+4 v T \sum_{q} \frac{1}{\tilde{r}+K q^{2}} .
$$

We can show that $\tilde{r}$ plays indeed the role of a surface tension by looking at the fluctuations of one Fourier component $u_{q}$. We find by similar methods

$$
\begin{aligned}
\left\langle\left|u_{q}\right|^{2}\right\rangle & =\frac{1}{q^{2}}\left\langle\left|m_{q}\right|^{2}\right\rangle \\
& =\frac{1}{q^{2}} \frac{T S_{0}}{\left(\tilde{r}+K q^{2}\right)} .
\end{aligned}
$$

This is exactly the form which is expected for a membrane of surface tension $\widetilde{r}$ (see for instance ref. [4]). According to our general arguments, we are led to impose $\tilde{r}=0$. In the phase transition analog, this means that we stand exactly at the transition point. There remains to show that there is a transition point, i.e. that eq. (2.22) with $\tilde{r}=0$ can be satisfied by a suitable choice of $r$

$$
r=-4 v T \sum_{q} \frac{1}{K q^{2}}=-\frac{4 v S_{0} T}{2 \pi K} \int_{q_{\min }}^{q_{\max }} \frac{\mathrm{d} q}{q}
$$

$q_{\max }=1 / a$ is some molecular cut-off. The lower cut-off $q_{\min }$ is imposed by the boundary conditions $q_{\min } \sim \pi / L$. Thus we are led to

$$
r=-\frac{E T}{4 \pi K}\left(\ln \frac{L}{a}+\text { const. }\right) .
$$

Note that the value of $r$ depends on the size of the membrane !

(This is typical of a two-dimensional spherical model, for which there is no transition in the limit $\mathrm{L} \rightarrow \infty$ ) [8]. A similar feature was already found in an appendix of ref. [1] where a weighting factor very similar to (2.14) was considered.

Numerically $T / K \simeq a / e$, where $e$ is a lipid layer thickness $(e \simeq 50 \AA)$. Thus $-r \sim E / 10 \sim$ a few $\mathrm{ergs} / \mathrm{cm}^{2}$. We conclude that it is possible to achieve the required equilibrium state, with $\tilde{r}=0$, provided that the unperturbed system has a certain tendency to increase its surface : this increase is then limited by the elastic forces.

Some readers may be surprised to find such a dramatic effect of fluctuations on surface tension parameters : the point is that these unswollen cells have huge fluctuations, much more important than in usual interfaces.

3. Membranes in contact with a reservoir. - Let us consider a black film with $N$ lipid molecules and a fixed surface $S_{0}$ (Fig. 1). The corresponding free enthalpy is of the form

$$
F=N \varphi\left(\frac{N}{S_{0}}\right)=N \varphi(\rho) .
$$

The function $\varphi(\rho)$ is minimal for a certain value $\rho^{*}$ of the surface density. It is important to realize that in 
the black film the equilibrium value of $\rho$ is not equal to $\rho^{*}$ : since the film exchanges molecules with the outer rim, which plays the rôle of a reservoir, we must in fact minimise the following potential

$$
G=N \varphi\left(\frac{N}{S_{0}}\right)+\mu_{\mathrm{r}}\left(N_{\mathrm{t}}-N\right)
$$

where $\mu_{\mathrm{r}}$ is the chemical potential of the lipid in the reservoir, and $\mathrm{N}_{\mathrm{t}}$ is the total number of lipid molecules (film + reservoir). The minimization condition gives a density $\rho_{0}$ defined by :

$$
\varphi\left(\rho_{0}\right)+\rho_{0} \varphi^{\prime}\left(\rho_{0}\right)=\mu_{\mathrm{r}} .
$$

Since the chemical neighbourhood of a lipid molecule in the reservoir differs from what it is in the film, we have $\mu_{\mathrm{r}} \neq \varphi\left(\rho^{*}\right)$ and eq. (3.3) gives $\rho_{0} \neq \rho^{*}$.

Let us now consider a different problem, where we start from an unperturbed system with area $S_{0}$ and $N_{0}=\rho_{0} S_{0}$, and then impose a small change in area :

$$
\left.\begin{array}{l}
S_{0} \rightarrow S_{0}+\delta S \\
N_{0} \rightarrow N_{0}+\delta N
\end{array}\right\} .
$$

The film stays in contact with the reservoir : thus eq. (3.3) remains valid and the density remains equal to $\rho_{0}$. This implies

$$
\delta N=\rho_{0} \delta S .
$$

The change in the potential $G$ is then

$$
\delta G=\delta N\left(\varphi\left(\rho_{0}\right)-\mu_{\mathrm{r}}\right)=-\rho_{0}^{2} \varphi^{\prime}\left(\rho_{0}\right) \delta S .
$$

There is a finite surface tension

$$
r=\frac{\delta G}{\delta S}=-\rho_{0}^{2} \varphi^{\prime}\left(\rho_{0}\right)
$$

Thus in a static light scattering experiment on a black film, we expect Fourier components of the displacement $u_{q}$ with mean square values of the form

$$
\left\langle\left|u_{q}^{2}\right|\right\rangle=\frac{T}{r q^{2}} .
$$

These static experiments are extremely hard to perform. Dynamic experiments have been realised recently on some black films [9], and they do suggest the existence of a small but finite surface tension. These experiments deserve some comments :

a) At finite frequencies, it is not certain that the local lipid density can indeed adjust itself to the optimal value $\rho_{0}$ : this depends on a delicate comparison between the frequencies of the capillary modes and of Lucassen modes [10]. However, this effect does not react on the restoring forces for capillary waves : for instance if, in a high frequency regime, $\delta N$ is zero (instead of being equal to $\rho_{0} \delta S$ ) the surface tension is $\partial F /\left.\partial S\right|_{N}$ and (as is clear from eq. (3.1) it is still equal to $r$ ).

b) The experimental surface tension is small (of order $1 \mathrm{erg} / \mathrm{cm}^{2}$ for cholesterol bilayers). One possible explanation of this point is sketched in figure 1 where we picture two possible states for the lipid molecule :

- at the rim (reservoir) the molecule is in contact with a deep octane fluid, very similar to its own aliphatic part,

- in the film there is very little octane : the short range interactions between one aliphatic tail and it's neighbourhood are not very different from the preceding case. But the long range interactions (the tail of the Van der Waals potential) are cut out because the film is thin.

It is this comparatively small effect which gives an equilibrium density $\rho_{0}$ (in the film) slightly smaller than $\rho^{*}$. The surface tension is proportional to $-\varphi^{\prime}\left(\rho_{0}\right) \cong \varphi^{\prime \prime}\left(\rho^{*}\right)\left(\rho^{*}-\rho_{0}\right)$ and is accordingly small.

4. Conclusions. - We have seen that the surface tension of thin membrane systems depends on certain specific conditions

4.1 For closed systems like unswollen red blood cells the static surface tension (after inclusion of all fluctuation effects) should vanish, and the fluctuations should be described by a very simple formula (derived from (2.23))

$$
\left\langle\left|u_{q}^{2}\right|\right\rangle=\frac{\dot{T}}{K q^{4}} .
$$

Thus, for static properties, we recover the simple predictions of the harmonic approximation in ref. [1].

Does this conclusion remain true for the dynamic experiments which probe the flicker ? We believe that it does, because for a given $q$ the frequency of the Lucassen mode [10], which relaxes the local density, is always much larger than the frequency of the peristaltic modes discussed in [1].

4.2 For open systems, we expect a finite surface tension, controlled by the differences in energy for one molecule in the film and in the reservoir. All these considerations may appear rather formal; they ignore the practical complications associated with red blood cell shapes, with their spontaneous curvatures, etc... But hopefully they should be of use as a starting point for more realistic discussions in many membrane systems.

Acknowledgments. - One of us (F. B.) has benefited from stimulating discussions with J. A. Cowen. 


\section{References}

[1] Brochard, F., Lennon, J. F., J. Physique 36 (1974) 1035.

[2] Mueller, P., Rudin, D., Titien, M. and Wescott, W. C., Nature 194 (1962) 979; J. Phys. Chem. 67 (1963) 534.

[3] For a complete review on $\mathrm{RBC}$, see :

BESSIS, M., Living blood cells and their ultrastructure (Springer-Verlag, Berlin) 1973.

[4] De Gennes, P. G., PaPoular, M., Volume jubilaire en l'honneur de A. Kastler (PUF, Paris) 1969.

[5] Toulouse, G. and Pfeuty, P., Introduction au groupe de renormalisation et à ses applications. Phénomènes des transitions de phase et autres (PUF, Grenoble) 1975.
[6] Evans, E. A., Biophys. J. 13 (1973) 926.

[7] Helfrich, W., Z. Naturforsch. 28c (1973) 693.

Helfrich, W. and Deuling, H. J., J. Physique colloq. 36 (1975) C 1-327.

[8] Berlin, T. H. and KaC, M., Phys. Rev. 86 (1952) 821. Barber, M. and Fisher, M., Ann. Phys. 77 (1973) 1.

[9] Grabowski, E., Cowen, J. A., to be published.

[10] Lucassen, J., Trans Faraday Soc. 64 (1968) 2221. 